\title{
The Condensation Process of Alcohol Molecules in the Nanochannel of MCM-41: A Spin-Probe ESR Study
}

Masaharu Okazaki, * Shinpei Iwamoto,\$ Yoshimi Sueishi,\$ and Kazumi Toriyama

Research Institute of Instrumentation Frontier,

National Institute of Advanced Industrial Science and Technology (AIST),

2266-98, Shimoshidami, Moriyama-ku, Nagoya, 463-8560, Japan,

\$ Department of Chemistry, Faculty of Science, Okayama University,

3-1-1, Tsushima, Naka, Okayama 700-8530, Japan.

masa-okazaki@aist.go.jp 


\section{SUPPORTING INFORMATIONS:}

I) Sample preparation method in detail:

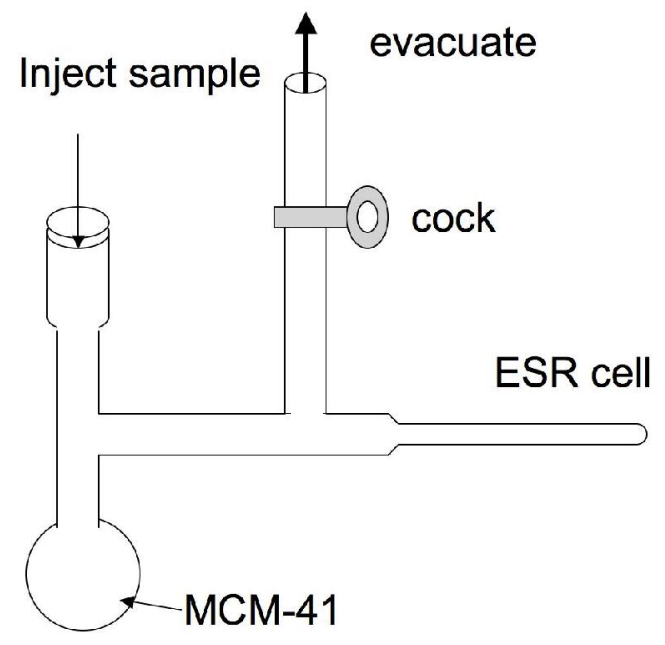

Figure 1S: glass ware for sample preparation and observation

MCM-41 was first dried in an oven at $403 \mathrm{~K}$ for about $1 \mathrm{hr}$ and the weight is precisely determined. After evacuating for another hour in a glass apparatus of Fig. $1 \mathrm{~S}$ at $403 \mathrm{~K}$ the cock is closed and the sample solution is injected with a micro syringe through the silicon septum with keeping the environment in vacuum. Since a very small amount of air may get into the apparatus during the process, the system is cooled by liquid nitrogen and evacuated once. The warmed sample is mixed vigorously to lead the solution distribution in equilibrium at this stage. Then the ESR spectrum is observed. The ESR spectrum is reproducible even after 24 hours. After the ESR observation another volume of solution is added and the above process is repeated. The vacuum keeping septum was obtained from Aldrich. 


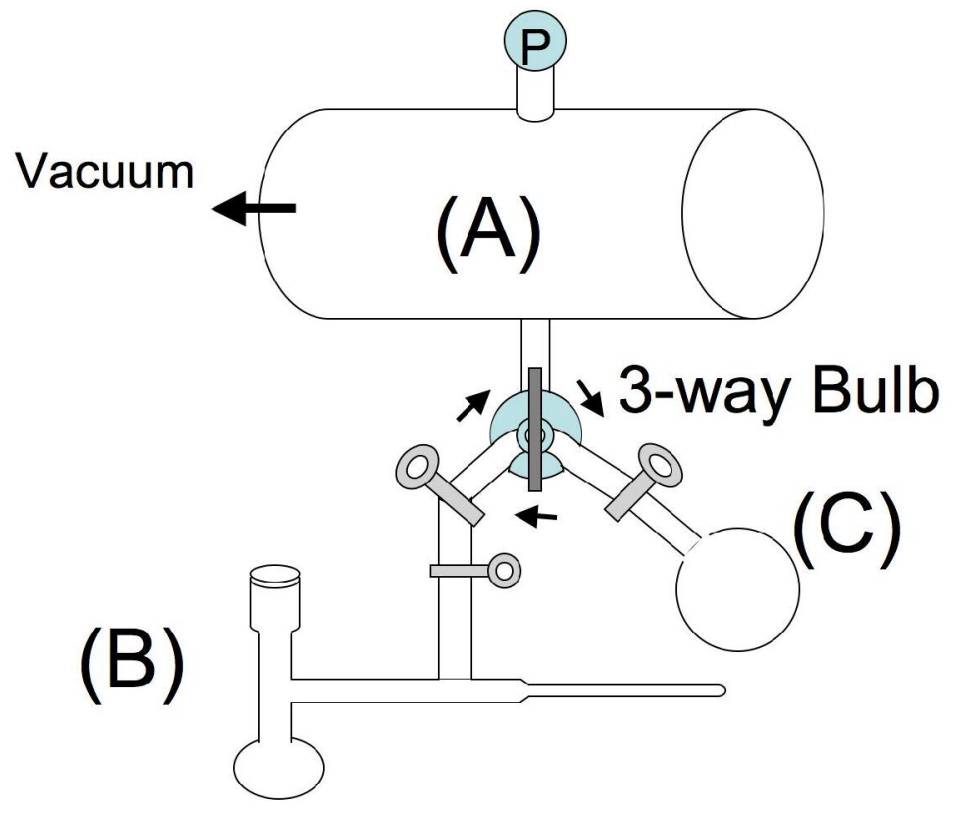

Figure 2S: Apparatus to introduce artificial air to the sample cell

The introduction of artificial air (mixture of $\mathrm{O}_{2}$ and $\mathrm{N}_{2}$ at the ratio of 1:4) to the system was made with the apparatus shown as Figure 2S. First, the artificial air was introduced to (A), whose volume was already measured, and then the three-way bulb is turned to connect (A) with (C). The volume of (C) can be determined from the pressure. (Baratron, MKS). After cooling the sample at $77 \mathrm{~K}$ in the spherical container of (B), the three-way bulb is turned to connect (B) with (C).

After evacuating (A) the three-way bulb is turned again to measure the gas left in container $(\mathrm{C})$ and the pressure is read with the gauge. The quantity of the artificial air in (B) can be calculated from the relative volumes and the pressure read in each step. The quantity of the gas is given formally as the pressure calculated from the volume of (B) (typically 10 $\mathrm{mL}$ ) without considering adsorption on the sample. This rather tedious method is employed since the alcohols have considerable vapor pressures. 


\section{II) ESR data for the Ethanol system:}

A)

B)

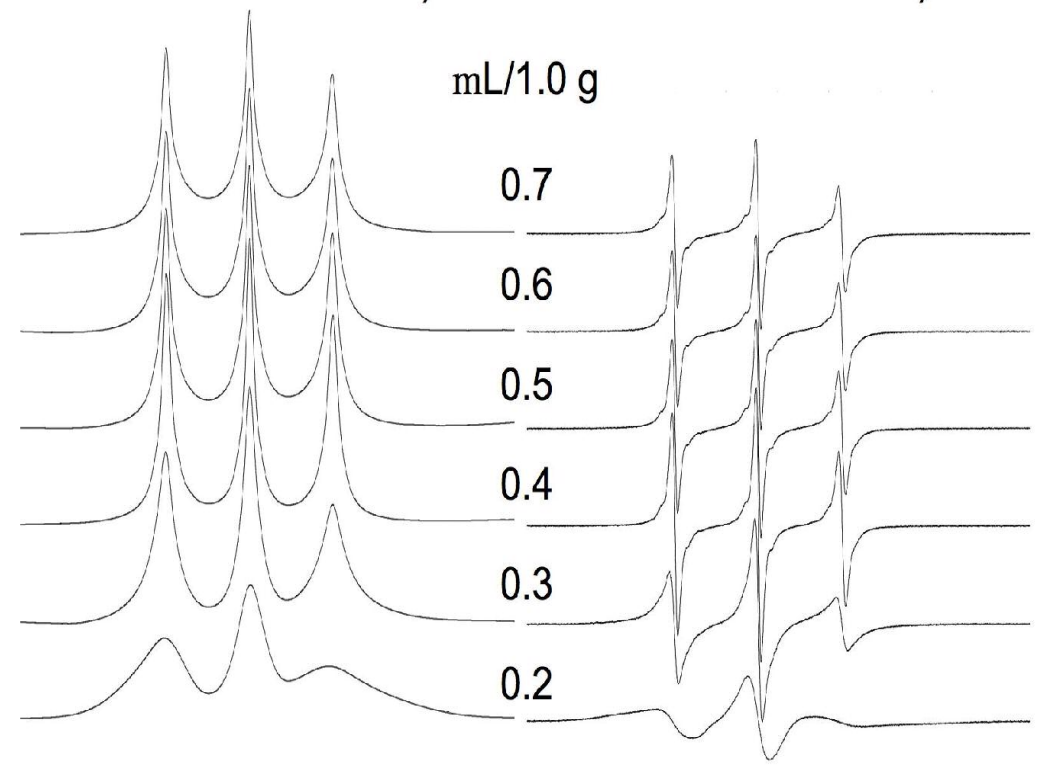

Figure 3S: DTBN solution of ethanol (30 mM) in MCM-41(12)

Figure $3 \mathrm{~S}$ shows the ESR spectra of the DTBN solution of ethanol condensed in the nanochannel of MCM-41(12) at various doses. 


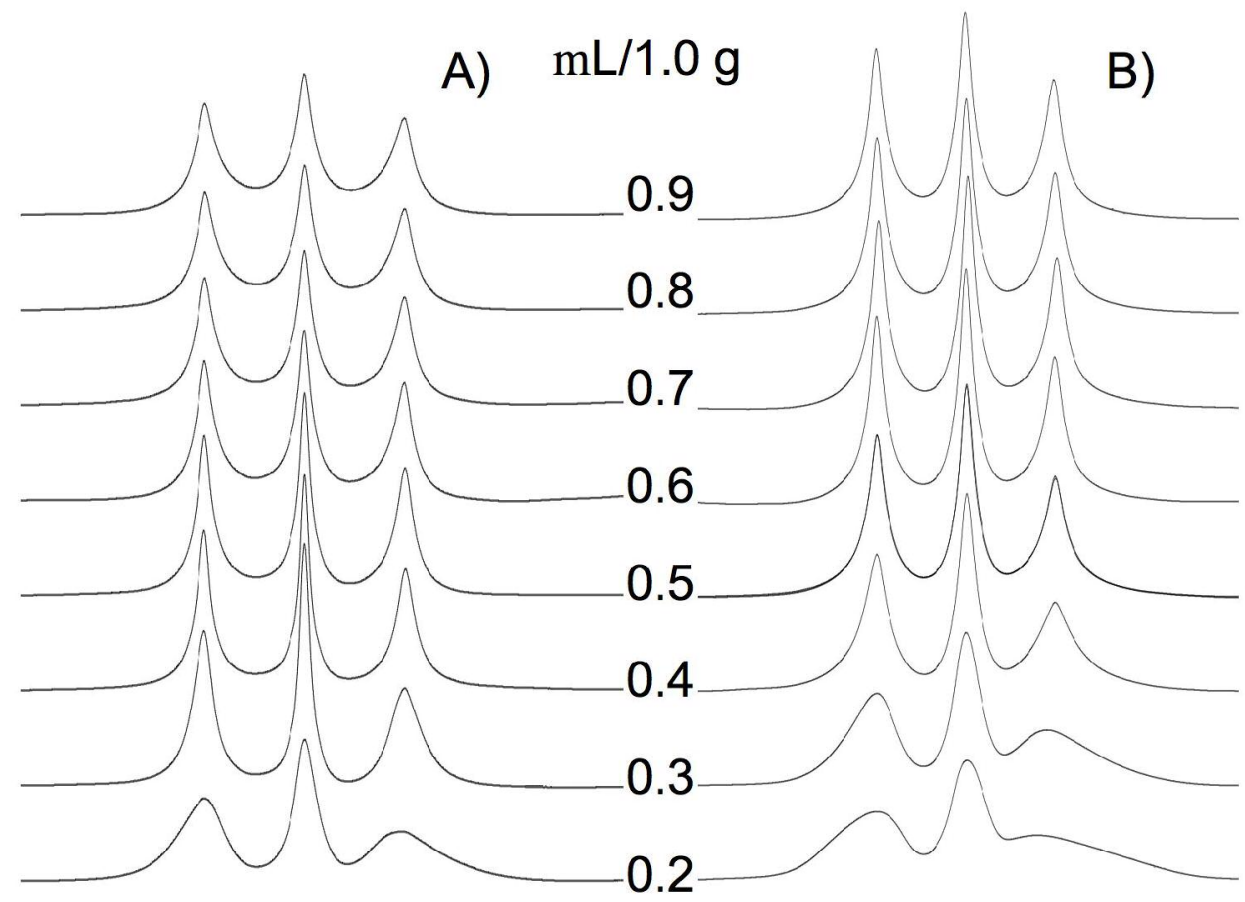

Figure 4S: Integrated version of Figure 4

The ESR spectra for the methanol solution (A) and for the 2-propanol solution (B) of DTBN at $30 \mathrm{mM}$ condensed in the nanochannel of MCM-41(16) at various doses. 


\section{$\mathrm{MeOH} / \mathrm{M}(10) 30 \mathrm{mM}$}

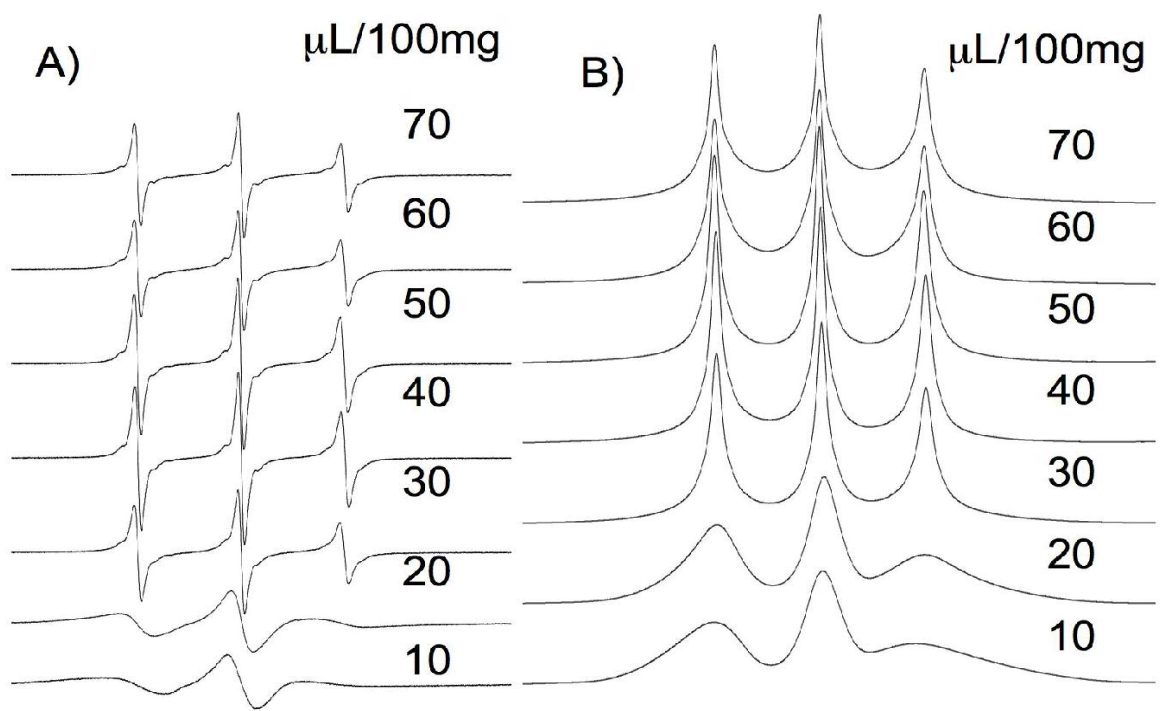

Figure 5S: ESR spectrum for a methanol solution of DTBN at $30 \mathrm{mM}$ condensed in the nanochannel of MCM-41(10) at various dose. The spectra in B) are normalized to give the same integrated value.

The most of the data on this system have been published in a previous report. ${ }^{14}$ The spectrum changes in a similar manner with the MCM-41(12) system. The linewidth (left column) at a dose higher than $0.3 \mathrm{~mL} / \mathrm{g}$ is smaller and the dose at which the signal height (right column) becomes maximum is higher compared with those for the MCM-41(12) system. These are due to the narrower channel diameter of MCM-41(10). The volume of the nanochannel has been estimated as $0.5 \mathrm{~mL} / \mathrm{g}$ from the spectrum patterns of column B) which is considerably smaller than the volume of $0.63 \mathrm{~mL} / \mathrm{g}$ calculated with the method given in Experimental. ${ }^{14}$ 